\title{
The Development of Computer Network and the Opportunity of Integration Automation
}

\author{
Huafeng Wei \\ China Coal Technology Engineering Group Chongqing Research Institute Co. Ltd, Jiulongpo, Chongqing, \\ China \\ renj1985@126.com
}

Keywords: Computer, Network, Integrated automation, Opportunity

\begin{abstract}
Under the background of rapid development of science and technology in China, computer network technology has also made a significant development. At this stage, how to promote the development of computer network towards integrated automation is a problem that scholars in this industry pay special attention to. This paper mainly analyzes the problems related to the development of computer network and the opportunities of integrated automation. The purpose of this paper is to conduct in-depth research on the development of computer network's integrated automation, and accelerate the development of China's integrated automation field.
\end{abstract}

\section{Introduction}

Social economy and science and technology directly affect the development of computer network and communication technology. In the history of our country, there have been many changes in computer network and communication technology. Researches on the computer network's integrated automation can make computer network and communication technology more conform to the trend of the development of the times, and can promote the progress of social economy and science and technology in China.

\section{The Development of Computer Network}

\subsection{Local Area Network (Lan)}

Computer network is a computer network communication system which connects more than two computers together through wireless communication technology and electric cable. At present, China's computer network can be divided into two forms according to their geographical locations: one is Local Area Network, the other is Wide Area Network. The so-called Local Area Network is a computer communication network composed of two or more computers, databases and external devices in a local geographical scope. Local Area Network is known as LAN for short. With the aid of special data circuit or data communication network, LAN is connected with remote LAN, data processing center or database to form an information processing system with larger coverage. LAN has many functions, such as file management, sharing applications and software, sharing printers, scheduling work, sending fax or e-mail, etc. LAN has a certain degree of exclusiveness, so it is widely used in office. Each computer or other network equipment on the LAN has one or more LAN IP addresses. The LAN IP addresses are assigned within the LAN. IP addresses of different LANs can be repeated and will not affect each other ${ }^{[1]}$.

\subsection{Wide Area Network (Wan)}

The so-called Wide Area Network (WAN) is what we often call the Internet. It is a network covering the whole world. Each computer or other network equipment on Wide Area Network (WAN) has one or more WAN IP addresses. WAN IP addresses can only be applied for after paying fees at ISP (Internet Service Provider). WAN IP addresses cannot be duplicated. Computers on WAN have to make use of the router or the NAT (Network Address Translation) of gateway ${ }^{[2]}$. 


\subsection{The Overall Development Stage of Computer Network}

The overall development of computer network includes four stages. The first stage is the embryonic stage of the formation of computer network, which began in the mid-1950s when the core of computer network is a single computer remote connecting system; the second stage is the primary stage of the formation of computer network, which started in the mid-1960s when the idea of host computer interconnection was formally proposed and single computers began to connect through multiple lines to form a computer network with a large coverage. But the network operating system has not appeared in this stage, so it can only be called communication network. In the mid-1960s, the second generation of computer network was officially born. The third stage began in the 1970s and 1980s, when Ethernet appeared and prevailed in the large market. At this time, OSI, the Open System Interconnection standard, was formally proposed and unified network structure was formed. However, it was necessary to strictly abide by international standardization protocol. Computers in this stage are called the third generation computer network. The fourth stage began in the mid-1990s when computer network kept the high speed and comprehensiveness as its development characteristics, and the multimedia and intelligent network has also been formed. Computers in this stage are called the fourth generation computer network, and the transmission speed of multimedia intelligent network is by Gigabytes.

\section{The Development of Integrated Automation}

Integrated automation is an important symbol of the development of science and also an effective measure for enterprises to innovate their production. Integrated automation mainly refers to computer software and hardware technology, instruments, electronic equipment, etc. With the rapid development of science and technology in China, the integration automation of large-scale system has gradually attracted people's attention. One of the most important contents of the Computer Integrated Manufacturing System is the system integration technology, which helps to improve the core competitiveness of enterprises in the market. At present, China's integrated automation technology includes many contents, such as information technology, field-bus technology, wireless technology, human-computer interface and security technology ${ }^{[3]}$.

\section{The Analysis on the Development of Computer Network and the Opportunity of Integrated Automation}

At present, enterprises' network framework with fieldbus as control layer and network as management and decision-making layer has been improved, which creates very favorable conditions for the development of computer network. For the integrated automation system, the working environment is better, and its own systematic integration can be further improved. In a word, the computer network technology in China is developing in the direction of both theoretical and technological control. The development of computer network and integrated automation opportunities can mainly be demonstrated in the following aspects: first, the display of internal information of the enterprise intranet can utilize the WWW information service technology, the application of which makes it convenient for the internal staff of the enterprise to reserve application information together. The information release of computer network technology has the advantages of simple-operation, innovation, independent platform and so on, which can achieve a wide range of system operation. The essence of corporation's Intranet is to reorganize and develop information and resources. The reorganized and developed information and resources can integrate the computer network environment, which is of great significance for the comprehensive promotion of decision support system and information management system.

The computer network technology developed in this form can break through the limitations of the original information management system, and can fully implement information services in the process of management and decision-making ${ }^{[4]}$. Second, for enterprises who want to improve their core competitiveness in the market and occupy a favorable competitive position, it is necessary to 
build a fieldbus distributed control system. The construction of fieldbus control system can have a positive impact on the distributed control system. For example, with the help of fieldbus intelligent instrument, a real distributed control system can be constructed. With the help of fieldbus intelligent instrument, PID and other control functions can be fully embodied to realize self-adaptive control or robust PID control. The distributed control system of fieldbus is based on the distributed control system (DCS) technology, which has occupied a certain position in industrial production automation and become a new research hotspot. In the 1990s, the fieldbus technology rose formally as an advanced industrial control technology. It introduces the concept of communication and management through network into industrial control. In essence, it is a digital communication protocol. It is a digital, fully distributed, two-way transmission and multi-branched communication network that connects intelligent field equipment and automation system. It is a combination of control technology, instrument industry technology and computer network technology. It has the technical characteristics of field communication network, field equipment interconnection, interoperable and distributed function blocks, Power Over Ethernet and open and interconnected network.These characteristics not only ensure that it can fully adapt to the needs of the industry for digital communication and automatic control, but also make it possible to interconnect it with the Internet to form a complex network of different levels, which represents a development direction of the industrial control system in the future. In a word, both intranet and fieldbus can change the traditional management structure of enterprise, making the enterprise management system more "flat" . Such kind of management mode can make the middle layer's function of planning and scheduling transfer to the upper and lower levels, and achieve the reorganization and hierarchical control of management functions. Under this background, in order to better meet the production requirements, enterprises began to develop application software and build their own software platform. The "platformization" and "flattening" in control theory are both opportunities and challenges for the development of integrated automation in computer networks ${ }^{[5-6]}$.

\section{Conclusion}

To sum up, in a new era when computer network is developing rapidly due to impact of social economy and science and technology, which has created good conditions for the birth of integrated automation, enterprises should take advantages of computer network and integrated automation system according to their actual needs, and constantly improve their competitiveness in the market.

\section{References}

[1] An Jun, "An Brief Analysis on the Computer Network And Opportunities of Integrated Automation”. China CIO News, no.9, pp.40, 2019.

[2] Li Biyan, "Research on System Integration and Integrated Automation System”. China Management Informationization, no. 14, pp. 59-61, 2011.

[3] Wei Xiaohao, "Design for the Integrated Intranet of the Assorted Computer Network for the South to North Water Transferring Project”, Small Hydro Power, no.2, pp. 32-34, 43, 2017.

[4] Xie Huibin, "The Application of Artificial Intelligence in Computer Network Technology". Computer Knowledge and Technology, vol.14, no.23, pp.231-232, 2018.

[5] Wu Mingde, "Analysis on the Application of the Computer Network in the Electric System". Digitization User, vol.23, no.33, pp. 172-173, 2017.

[6] Li Hui, He Li., Chen Lugen, et al. "On the Development and Application of Self-evaluation System for Mine and Gas Samples Based on the Network Integration Technology". Coal Technology, vol.38, no.7, pp.141-143, 2019. 